\title{
Problemática actual de la población de Huesca
}

\author{
M. ${ }^{a}$ Teresa Rubio Benito *
}

\section{APROXIMACION AL TERRITORIO}

Todo el territorio aragonés acusa el problema generalizado del despoblamiento rural. Aragón se define por sus grandes vacíos demográficos y por su economía insuficientemente desarrollada, con fuertes desequilibrios espaciales. La evolución de la población aragonesa desde principios del siglo actual hasta 1981, ofrece un panorama desolador en las provincias de Huesca y Teruel en continuo descenso, la primera, y con caída prácticamente irrecuperable, la segunda, a partir de 1950, aún cuando la línea regional que hace de bisectriz del ángulo en relación a la población nacional, enmascare la realidad, por el tirón hacia arriba que supone Zaragoza capital, ciudad dinámica que supera los 600.000 habitantes y sigue en alza por su privilegiada posición de llave del Ebro en el triángulo Barcelona-Pais VascoMadrid, como una isla en medio de su propia provincia despoblada y de los vacíos turolenses y oscenses.

Huesca y su provincia por lo tanto, no constituyen excepción, más bien al contrario, se hallan inmersas en una situación de desarticulación socioeconómica grave.

Situada al Norte del territorio de la Comunidad Autónoma de Aragón, con algo más de $15.000 \mathrm{~km}^{2}$ de extensión, Huesca representa el 33 por 100 del territorio regional y el 3 por 100 del total nacional.

- Profesora Titular de Geografia Humana UNED 
El último Censo (1981) arrojaba una cifra de 219.813 habitantes, lo que supone una densidad de $14 \mathrm{~h} / \mathrm{km}^{2}$, repartidos de forma muy desigual en la geografia provincial de grandes contrastes latitudinales: altos valles pirenáicos entroncados en el centro de la cadena montañosa, extensos somontanos y llanuras de piedemonte, enlazados con los llanos que dan paso a la depresión media del Ebro. (J. L. CALVO PALACIOS, 1981).

La población se asienta de forma preferente en el área formada a caballo de la arteria principal de comunicación de carretera (la nacional 240) o en sus inmediaciones, quedando englobados en su perímetro las ciudades de la provincia con mayor densidad de población que centran a su vez las principales actividades agricolas, industriales y de servicios, consecuencia de las grandes obras de regadíos, del establecimiento de polígonos y zonas de preferente localización industrial y de las derivadas del tráfico comercial, transportes y otros servicios complementarios.

\section{EL CARACTER REGRESIVO DE LA POBLACION}

Desde principios de siglo hasta la actualidad, el contingente de población de la provincia de Huesca es claramente regresivo, con un porcentaje de disminución del 12 por 100 debido a la corriente migratoria que repercute también en el comportamiento de las tasas de natalidad y mortalidad. La natalidad ha disminuido de forma alarmante en Aragón, oscilando cinco puntos por debajo de la media nacional (M. MOLINA IBAÑEZ y J. M. MARIN JAIME, 1981).

La provincia de Huesca tiene unas tasas de natalidad muy bajas y siempre inferiores al resto de Aragón y a la media nacional, hecho que vendría ocasionado (J. A. BIESCAS, 1977) por la persistencia de instituciones jurídicas tales como el mayorazgo, que al hacer que todos los bienes familiares pasen a manos del heredero, genera una situación social en la que los otros hermanos están abocados a seguir trabajando en el patrimonio familiar sin disponer de bienes, lo cual va a impedir, o al menos dificultar, que contraigan matrimonio. 
Hace diez años la tasa de natalidad en la provincia de Huesca era del 11,58 por 1.000 y la de mortalidad 10,23 por 1.000 de tal forma que el crecimiento vegetativo se situaba en un 1,35 por 1.000 , ocho puntos por debajo de la tasa de crecimiento de España y cuatro puntos por debajo de la de Aragón. En la actualidad, el envejecimiento progresivo de la población es la tónica generalizada. Los datos de 1983 sitúan en 9,82 por 1.000 la tasa de mortalidad. El decrecimiento de la natalidad al 9,63 por 1.000 va a llevar a una dinámica demográfica regresiva que puede hipotecar el desarrollo provincial. En efecto, el crecimiento demográfico provincial viene siendo negativo en la provincia de Huesca desde el siglo pasado como podemos constatar con el cuadro I de la obra «Población y territorio en España ss. XIX y XX” (RODRIGUEZ OSUNA, 1985), en el que Huesca aparece sistemáticamente incluida dentro de las provincias con saldo negativo, hecho éste atestiguado con mayor detalle en la evolución censal del período comprendido entre 1877 y 1981, en el que observamos (cuadro II) un máximo de población de 255.177 habitantes, alcanzado en 1887, para descender seguidamente, con algunos pálpitos de recuperaciones breves a principios de siglo, sobre todo en los años 20 , en que el índice se sitúa en 99 debido quizá al retorno de emigrantes cuyo destino habían sido países beligerantes en la primera Guerra Mundial (F. QUINTANA, 1981) o a la llegada de la segunda oleada de innovaciones industriales (el ciclo electricidad-química-sintéticos) que penetró en España en los años 20 aunque no se completó hasta los 50 y 60 debido a la guerra civil y que afectaría a algunos puntos del Norte de la provincia relacionados con el sector eléctrico. De cualquier forma, el efecto fue poco duradero, cayendo a continuación en picado con una pequeña recuperación en 1950, época que coincide con las construcciones de pantanos en la provincia que atrajeron mano de obra joven de otras regiones españolas.

\section{INFRAESTRUCTURA VIARIA Y CRECIMIENTO DE LA POBLACION}

En contraste, la capital, mantiene un ritmo de crecimiento continuo, lento, más o menos de un punto, mantenido en torno a los 15.000 habitantes hasta después de la guerra civil y será a partir de 1950 y sobre todo de 
M. ${ }^{a}$ TERESA RUBIO BENITO

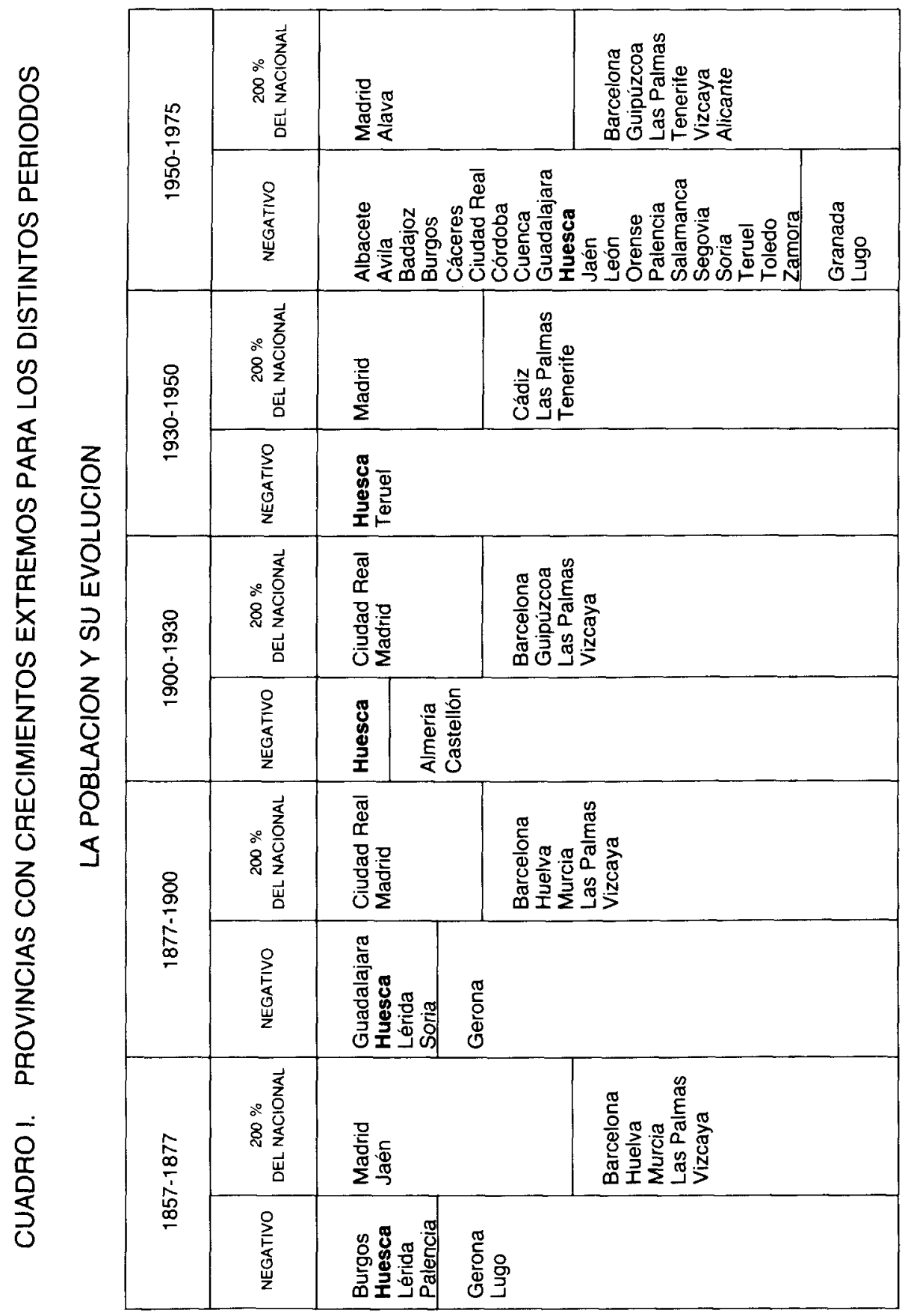

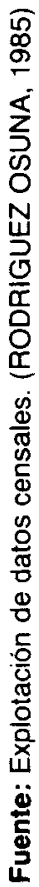




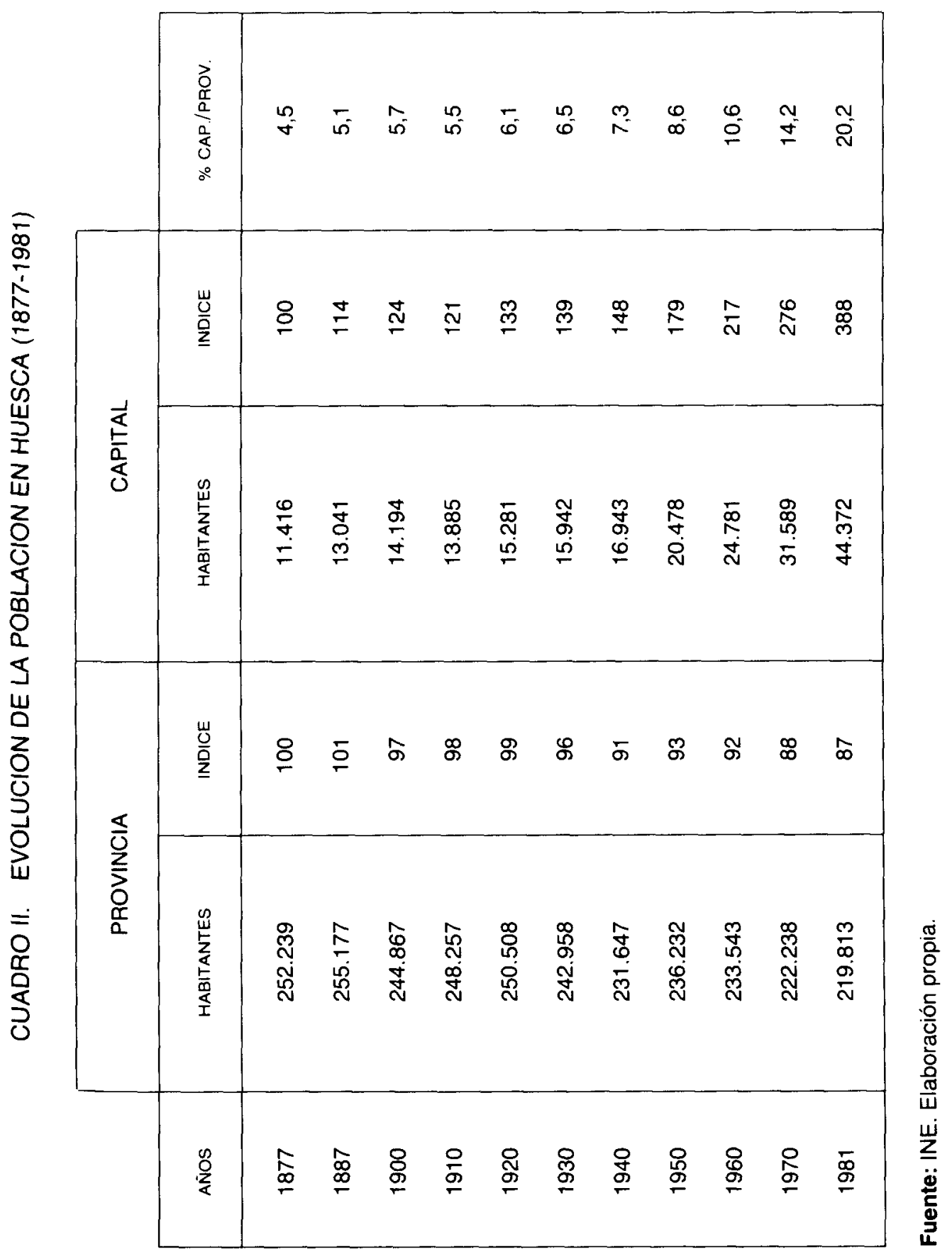


1960, cuando se dispararán las cifras hasta duplicarse la población en la ciudad, a la par que se despuebla la provincia de un modo selectivo ya que afecta fundamentalmente a las comarcas más pobres y peor comunicadas, marcadas por el impacto de la emigración. Huesca, la capital, junto con Sabiñánigo, Barbastro, Monzón, Fraga, Ainsa y Sallent, constituirán auténticos islotes de poblamiento generados por el dinamismo económico de sus establecimientos industriales, en algunos casos o por el desarrollo del terciario en otros. (M. MOLINA IBAÑEZ, y J. M. MARIN JAIME, 1981). Aunque en ambos supuestos siempre estarán implicitas unas buenas comunicaciones, imprescindibles para cualquier actividad socioeconómica. El mapa de comunicaciones se identifica con las unidades de relieve, adaptándose el trazado de las carreteras, a los grandes surcos abiertos por los rios pirenaicos, cortando transversalmente la cordillera en amplios valles que alcanzan la depresión intermedia de la Canal de Berdún, la Val Ancha y otras depresiones interiores paralelas al Pirineo (págs. 213 y 214).

La dureza del relieve y las condiciones climáticas adversas han reforzado la pasividad de la Administración en cuanto a la creación de una infraestructura viaria adecuada, de forma que de cada cuenca fluvial, sólo se sigue una vía de acceso sin que se haya creado una auténtica red más o menos tupida, que asegure las comunicaciones entre los núcleos de población; más bien al contrario, existen auténticos vacíos que posibilitan e incluso fuerzan el despoblamiento, como es el caso de grandes zonas de las comarcas de Sobrarbe y Ribagorza o la amplia zona que comprenden las sierras exteriores surcadas por un dédalo de tortuosas carreteras comarcales en el mejor de los casos. Sólo el Bajo Cinca, el Somontano y la Hoya de Huesca, tendrán asegurados su comunicación y por tanto, su desarrollo a través de la carretera nacional 240 que como hemos dicho anteriormente, constituye el eje de desarrollo provincial sobre el que se asientan cinco ciudades que superan los 10.000 habitantes y la capital. Se trata de Jaca, Sabiñánigo, Huesca, Barbastro, Monzón y Fraga; todas ellas excepto Jaca, cuentan con polígonos industriales de reciente creación aunque su tradición industrial se remonte a principios de siglo.

Destacan las industrias básicas y ello como consecuencia de la producción de electricidad localizada en la orla pirenáica, la química, por los establecimientos industriales de Sabiñánigo y Monzón, y por último las metálicas básicas por la producción de aluminio ubicada en Sabiñánigo. 


\section{EL EQUILIBRIO DE LAS CIUDADES INTERMEDIAS}

Son estas ciudades citadas, las de mayor peso en el sector industrial provincial y aquellas en donde se produjeron hace pocos años, los trasvases humanos más espectaculares campo-ciudad. En Sabiñánigo, la utilización del espacio para usos industriales es un hecho relativamente reciente, ya que data del primer tercio del siglo actual.

El agua y su aprovechamiento como generador energético, será el elemento clave de transformación del suelo agrícola en industrial, centrándose esta actividad exclusivamente en Sabiñánigo.

Inicialmente, la construcción de obras hidráulicas se vinculó a la nécesidad de poner en regadío considerables extensiones de hectáreas, aprovechando para ello las tres grandes cuencas fluviales de los ríos Aragón, Gállego y Cinca en una región donde el déficit de precipitaciones y la sed son endémicos. Las asociaciones de regantes, las Cámaras Agrarias y la Confederación Hidrográfica fueron acicates para la acción del Gobierno sobre el desarrollo de la política hidráulica general y regional.

Cuando aumentaron las necesidades energéticas ya no bastó el uso del agua polarizado únicamente para el riego, sino que aprovechando los saltos y desniveles topográficos existentes se contó con la producción de energía hidroeléctrica.

Era el primer paso para asegurar un desarrollo apoyado en las vías naturales de comunicación que cruzan el Valle del Ebro con Francia.

El espectacular crecimiento de Sabiñánigo, que ha pasado en lo que va de siglo, de su condición de lugar con 280 habitantes a ciudad de 9.538 habitantes, según el Censo de 1981, tiene esta explicación. La construcción de una estación de ferrocarril a principios de siglo y la instalación de pequeños centros comerciales y de servicio en torno a la misma, dieron lugar a la creación de un núcleo compacto de población situado a $1 \mathrm{~km}$. del núcleo primitivo, constituyendo un barrio con entidad propia que pronto multiplicó sus efectivos poblacionales con la instalación de empresas industriales de capital extranjero atraído por la facilidad y bajo coste de la energía y por la accesibilidad al ferrocarril. 
La primera empresa instalada en Sabiñánigo fue Energia e Industrias Aragonesas, sociedad constituida en abril de 1918 con un capital de tres millones de pesetas, aportado mayoritariamente por un grupo francés relacionado con empresas de electroquímica, aluminio e hidroelectricidad, que puso en funcionamiento, en 1923, la primera fábrica de obtención de amoníaco a partir del hidrógeno electrolítico, complementando, dos años más tarde estas instalaciones, con industrias de clorato, ferromanganeso, explosivos y carburos (M. T. RUBIO BENITO y A. CASTELLÓ PUIG, 1986).

La Sociedad de Aluminio Español que comienza a funcionar en 1925, también con capital francés, transformando la bauxita del país vecino, representa en la historia de la industrialización de Sabiñánigo en el valle del Gállego, un jalón importante en la transformación de la actividad económica de la zona circundante y en definitiva del paisaje. Al inicio de los años treinta, se había producido ya una ampliación de la producción y del capital, controlado para entonces por el Banco Urquijo al igual que la empresa E.I.A.S.A. La clave del éxito radicaba en buena medida en la obtención de una energía barata aprovechando los vasos naturales y el desnivel existente entre los mismos. Con nuevos saltos de agua, se refuerzan las industrias existentes y se generan otras nuevas; en este sentido hay que señalar las ampliaciones realizadas por Energia e Industrias Aragonesas S. A. para intensificar sus fabricaciones clásicas de productos electroquímicos, proporcionando, además, la base para que se establecieran como filiales, una serie de empresas como: “Desarrollo Químico Industrial, S. A. (DEQUISA), Fósforos del Pirineo, S. A. y Aragonesas Promoción de Obras y Construcciones, S. A. y a partir de los años sesenta, en colaboración con la Junta de Energía Nuclear, se montaron las instalaciones necesarias para la recuperación del agua pesada, procedente de las baterias de electrolisis, convirtiéndose de esta forma en empresa avanzada en el campo de la energia nuclear.

Por su parte, Aluminio Española incrementó su producción de aluminio y estableció la gran planta manufacturera de Aluminio Transformación, S.A. y de Earle Aluminio Español, S. A., con fuertes inversiones que más tarde pasaron a denominarse Aluminio de Galicia, sociedad en la que sigue siendo mayoritario el capital francés, ahora en manos de la multinacional Pechiney. Entre los años 50 a 70, Sabiñánigo vivió un progresivo desarrollo industrial, creándose más de un millar y medio de puestos de trabajo ocupados fundamentalmente por vecinos del entorno próximo, bien de los municipios ubicados en el eje del Gáliego, bien en el de sus afluentes, como el río Basa y el río Guarga, manteniendo a veces en los mismos una agricultura a tiempo parcial, actividad que fue definitivamente abandonada dos años más tarde, de tal forma que bastantes municipios dentro del área de influencia de Sa- 
biñánigo, han quedado totalmente despoblados o con una población a punto de extinguir.

En julio de 1981 y para reforzar las enormes posibilidades industriales de esta zona, se creó en la misma el último polígono industrial de la provincia de Huesca, con una extensión de unos $413.660 \mathrm{~m}^{2}$ de suelo urbanizable, de los que aproximadamente $306.245 \mathrm{~m}^{2}$ son de suelo industrial y 90.655 $\mathrm{m}^{2}$ de viarios (pág. 215).

En Monzón el crecimiento espectacular de la población fue posterior al de Sabiñánigo ya que el despegue arranca de la década de los 50 a los 60 . En 1930 contaba con poco más de 4.000 habitantes mientras Barbastro en aquellas fechas, duplicaba, por asi decirlo, la población de Monzón. El inicio del cambio comenzará a notarse en la década de 1950, en que Barbastro mantiene su población prácticamente igual, mientras Monzón sube 1.000 habitantes, gracias a la instalación en 1946 de la primera factoría Hidro-Nitro Española, S. A. que comenzaba a dar sus frutos, compensando en parte el saldo negativo arrojado por la comarca de Barbastro entre 1901 y 1930, período en el que García Barbancho califica como espectacular la emigración de unos 13.000 habitantes debido a la ruina provocada por la filoxera y al comienzo de la mecanización (M. T. RUBIO BENITO, 1983).

Coincidiendo con una década negativa de Barbastro que se sitúa en 1960 con 10.227 habitantes, el avance de Monzón en este Censo es impresionante, llegando casi a doblar su población con 9.190 habitantes, alcanzando 14.600 en la década de los 70 .

Efectivamente, la instalación de Hidronitro en 1946, de su industria auxiliar de cementos, de Etino-Química 1950 (después Monsanto-Aiscondel) y pequeños focos metalúrgicos, provocó un crecimiento desmesurado de la demanda en el mercado de trabajo local. Entre 1940 y 1970, la tasa de incremento de población fue del orden del 204 por 100, mientras que el conjunto de la provincia sufrió una recesión del $-4,06$ por 100 . Este crecimiento, acentuado sobre todo desde la década de los 60 , refleja un periodo con altas tasas inmigratorias y elevados índices de movilidad (12\%). El incremento de la población de Monzón desde principios de siglo hasta el censo de 1981 era del 288 por 100, siendo más acusada en el período transcurrido desde 1940 hasta $1981(219 \%)$, trabajando en el sector secundario más de 3.000 personas. Mientras tanto, la emigración rural supuso para Huesca la pérdida en sólo tres años (1962-1965) del 25,33 por 100 de su población activa agraria. 
La atracción e instalación en Monzón de otras industrias llevó a la creación del polígono industrial «Paules» en 1970 con una superficie de $420.000 \mathrm{~m}^{2}$. No obstante haber señalado la importancia de Sabiñánigo y Monzón como centros industriales destacables por encima del nivel provincial, la proximidad de Barbastro al último centro señalado, hace que formen un tándem que junto con Fraga constituyen un frente oriental de atracción o al menos de fijación de población, de primer orden. El polígono de Barbastro construido por el INUR en 1974 , tiene una superficie de $933.000 \mathrm{~m}^{2}$.

\section{LA DISMINUCION DEL NUMERO DE MUNICIPIOS}

Fuera del eje de desarrollo señalado, puesto nuevamente de manifiesto en el mapa que refleja el Censo de municipios de 1970, la población se reparte en multitud de pequeños asentamientos de 200 habitantes. Hay que señalar que tan sólo 66 municipios superan los 500 de un total de 254 para este Censo y que 12, incluida la capital de la provincia, sobrepasan los 3.000 , manifestándose una clara tendencia generalizada a la pérdida de población respecto al Censo de 1960. Tan sólo aquellos municipios que estén situados cerca del eje de desarrollo o los promocionados por el impulso turístico, como algunos en el Valle de Tena, aumentarán población, aunque débilmente, como podemos comparar en el Censo siguiente. Efectivamente, en 1981, continúa la pérdida de población afectando no sólo a núcleos pequeños (los de más de 500 habitantes bajan a 55) sino también a los que mantenian una población superior a los 3.000 habitantes, como puede ser el caso de Graus, Monzón, Tamarite, Almudévar y Grañén, que bajan a 10 de tal forma que los dos últimos señalados pierden su condición de municipios con más de 3.000 habitantes. lgual ritmo puede apreciarse en los municipios de más de 500 habitantes. En el Pirineo, tan sólo Panticosa y Aísa se mantienen en aumento por el impacto producido en su economía por las estaciones de nieve de Panticosa y Candanchú, respectivamente.

Mientras tanto, sigue extendiéndose hacia el E. la mancha de despoblamiento en el $\mathrm{N}$. de la provincia, afectando de lleno a la comarca de Sobrarbe y también, aunque en menor grado, a la de Ribagorza, manteniéndose la zona de Monegros prácticamente estable con pérdida de población en Valfarta respecto al Censo anterior. 
Utilizando el concepto de municipio en su sentido más amplio —uno o varios núcleos de población dentro de unos límites administrativos- y ateniéndonos a las cifras que proporcionan los respectivos censos, actualmente se contabilizan en Aragón 724 demarcaciones correspondiendo 291 a Zaragoza, 233 a Teruel y 200 a Huesca.

La política de anexiones y fusiones llevada a cabo por la Administración local, junto con el proceso de despoblación de entidades enteras, han desembocado en la situación antes descrita que, por supuesto no tiene mucho que ver con la de principios de siglo. Por aquellas fechas, el cómputo más elevado correspondía a Huesca con 362 municipios, seguido de Zaragoza con 306 y Teruel con 279. Las pérdidas, por lo tanto, en orden decreciente han oscilado entre el 44,8 por $100,16,5$ por 100 en el caso de Teruel y 5 por 100 en Zaragoza.

Como en tantos otros campos, los años sesenta han marcado una barrera que separa el antes y después en el fenómeno de la despoblación. Hasta esas fechas, Huesca aportaba el mayor porcentaje de municipios de Aragón, pero fue descendiendo paulatinamente, en términos relativos, desde el 38,2 por 100 en 1900, hasta el 31 por 100 en 1970, fecha en la que su aporte a la región es inferior al de las otras dos provincias - 36,3 en Zaragoza y 32,7 por 100 en Teruel- (ver cuadro III).

No obstante, a nivel provincial, el declive ya se vislumbra a partir de los años treinta, pues mientras en Zaragoza la reorganización de municipios sólo supone una variación de un punto, y en Teruel, incluso aumenta - lo cual no indica aumento de población-, en Huesca la pérdida es progresiva desde los años treinta en que empieza a tener alteraciones en un 2,5 por 100 , continuando cada década con variaciones negativas, que en 1970 suponen un 30 por 100 de pérdida respecto a principios de siglo y un 44,8 por 100 en 1981 .

Pero si bien esta disminución en número nos pudiera hacer concluir que algunos municipios han aumentado de población, la realidad nos demuestra que no es así. $Y$ mucho menos si descendemos al nivel de entidades singulares o núcleos menores, donde el descenso ha sido considerable, hasta llegar a la despoblación total. La cuestión es que unos pocos han crecido por la desaparición real o administrativa de muchos. 
M. ${ }^{a}$ TERESA RUBIO BENITO

\begin{tabular}{|c|c|c|c|c|c|c|c|c|c|c|}
\hline \multirow{3}{*}{ 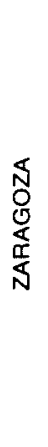 } & 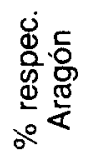 & m & लू & Ñ & $\begin{array}{l}\text { N } \\
\text { N్ }\end{array}$ & $\begin{array}{l}\text { ¿ें } \\
\text { ले }\end{array}$ & $\begin{array}{c}0 \\
\text { Ñ }\end{array}$ & న్ & $\begin{array}{l}m \\
\text { é }\end{array}$ & $\begin{array}{l}N \\
\text { O }\end{array}$ \\
\hline & $\alpha^{\circ}$ & 음 & 8 & $\hat{\operatorname{s}}$ & $\hat{\sigma}$ & $\hat{\sigma}$ & $\hat{\sigma^{\prime}}$ & 官 & $\stackrel{\Delta}{\sigma}$ & مீ \\
\hline & $\stackrel{\circ}{=}$ & ஜ্ల & ஜ্ল & ల్ల & 용 & 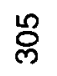 & ల్ల & ষ্ঠ & $\stackrel{\infty}{\stackrel{N}{N}}$ & $\overline{\mathbb{N}}$ \\
\hline \multirow{3}{*}{ 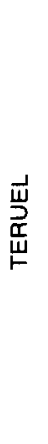 } & 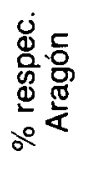 & 吕 & $\stackrel{n}{\mathscr{N}}$ & $\begin{array}{l}10 \\
\text { S }\end{array}$ & S) & S) & $\bar{\varnothing}$ & 요 & స్లి & $\begin{array}{l}N \\
\text { N }\end{array}$ \\
\hline & ¿ & 요 & 음 & 으 & $\overline{0}$ & 5 & 으 & $\overline{0}$ & $\mathscr{8}$ & $\stackrel{\infty}{\infty}$ \\
\hline & $\stackrel{\circ}{c}$ & 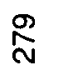 & $\underset{N}{\stackrel{9}{N}}$ & $\stackrel{9}{\stackrel{9}{N}}$ & N & $\stackrel{\sim}{\stackrel{N}{N}}$ & $\stackrel{\mathbb{N}}{\stackrel{N}{N}}$ & $\stackrel{\sim}{\stackrel{N}{N}}$ & 㣽 & $\stackrel{\mathscr{N}}{N}$ \\
\hline \multirow{3}{*}{ 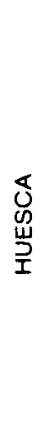 } & 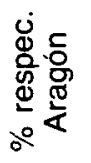 & $\begin{array}{l}\underset{N}{\infty} \\
\stackrel{\infty}{\infty}\end{array}$ & $\begin{array}{l}\underset{N}{\infty} \\
\stackrel{\infty}{\infty}\end{array}$ & $\begin{array}{l}\stackrel{\sim}{~} \\
\stackrel{m}{m}\end{array}$ & $\stackrel{\infty}{n}$ & $\hat{n}$ & $\stackrel{m}{m}$ & $\frac{m}{m}$ & $\frac{0}{m}$ & $\stackrel{o}{N}$ \\
\hline & ஃ゚ & 8 & 요 & 8 & $\begin{array}{l}\mathscr{0} \\
\stackrel{\infty}{\infty}\end{array}$ & $\begin{array}{l}\circ \\
\text { ó }\end{array}$ & 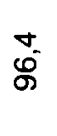 & 苑 & $\bar{R}$ & $\begin{array}{l}\stackrel{v}{n} \\
\stackrel{n}{n}\end{array}$ \\
\hline & $\stackrel{\circ}{\circ}$ & @్ల & @్ల & 心్ల & 命 & 足 & 昌 & 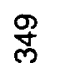 & 芯 & 유 \\
\hline & 号 & 용 & $\frac{ㅇ}{\sigma}$ & 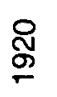 & $\begin{array}{l}\stackrel{\rho}{\text { }} \\
\stackrel{-}{-}\end{array}$ & $\begin{array}{l}\text { 염 } \\
\text { क }\end{array}$ & $\begin{array}{l}\text { 윰 } \\
\text { م }\end{array}$ & $\begin{array}{l}\stackrel{8}{\circ} \\
\stackrel{-}{-}\end{array}$ & 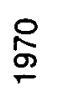 & $\underset{\infty}{\mathscr{\infty}}$ \\
\hline
\end{tabular}




\section{LOS CONTRASTES ESPACIALES EN LA DENSIDAD DE POBLACION}

La baja densidad de población (14 hab./ $\mathrm{Km}^{2}$ ) a nivel general, sugiere unos efectivos demográficos poco vitales, sin capacidad de reacción, si observamos que la situación se mantiene imparable desde hace más de un siglo. El mapa de densidad de la población en 1981 pone de manifiesto lo que ya hemos comentado, es decir, que las más altas se sitúan de la mitad de la provincia hacia el Sur y más concretamente hacia el S.E., manteniendo por supuesto más de $16 \mathrm{hab} . / \mathrm{Km}^{2}$ todo el eje desarrollado de la Nacional 240 , si bien queda patente el despoblamiento del área que organiza Sabiñánigo y que arrastra también a Biescas, producido en el Valle de Serrablo, Valle de Guarga y Valle de Nocito, fundamentalmente y en la crisis del reajuste industrial que afecta a Sabiñánigo como a otras áreas industrializadas desde 1972 debido a la crisis mundial de la enegía.

Los vacios de menos de $7 \mathrm{hab} . / \mathrm{Km}^{2}$ dejan prácticamente como una isla a Jaca y Sabiñánigo en toda la mitad Norte de la provincia, enmascarando muchas veces una realidad más dura ya que la cifra de 7 hab. $/ \mathrm{Km}^{2}$ puede considerarse holgada en muchos municipios que no superan los $3 \mathrm{hab} . / \mathrm{Km}^{2}$ e incluso menos.

Coinciden estos vacios como puede apreciarse, con los altos valles pirenaicos y todavia más duro o más acusado, con la zona de Sierras Exteriores, aunque no faltan tampoco en Monegros con carácter más matizado que en el Norte.

Las tres grandes comarcas al Norte de la provincia, Jacetania, Sobrarbe y Ribagorza, acusan pérdidas importantes de población, muy especialmente estos dos últimas, auténticas bolsas de despoblamiento (pág. 216).

El hecho de tratarse de áreas desfavorecidas o deprimidas, con economía exclusivamente agraria a veces complementada con un aprovechamiento silvopastoril, ha hecho que desde antiguo, los altos valles pirenáicos mantuvieran una densidad de población extremadamente débil y prácticamente estacionaria hasta la década de los 50 , en la que la corriente migratoria se acelera decididamente hasta convertirse en un proceso irreversible de desertización y envejecimiento a partir de 1960. (Ver cuadro IV.) 
M. ${ }^{a}$ TERESA RUBIO BENITO

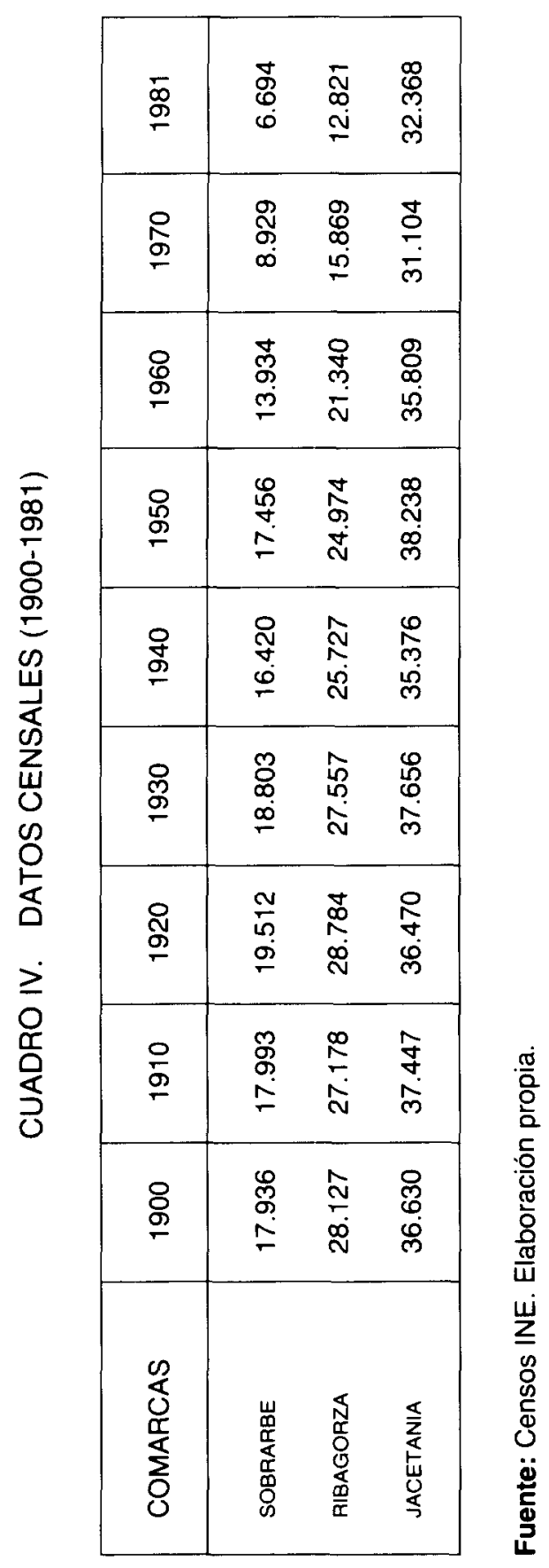


Según los efectivos de población que arrojan los censos, el Oeste de las tres zonas objeto de estudio, la Jacetania, mantiene un ritmo de descenso, en cifras absolutas, más contenido que el de Sobrarbe y Ribagorza, observándose dos máximos censales: el de 1930 que arroja un total de 37.656 habitantes, indicativo de la evolución natural de la población, y el de 1950 que -alcanza la cifra más alta en términos absolutos en lo que va de siglo, con 38.238 habitantes, cifra que recoge los nacimientos diferidos por causa de la guerra civil y el refuerzo de efectivos militares con sede en Jaca, compensando asi temporalmente el bache poblacional que sufrieron los años de contienda y que viene reflejado en el censo de 1940. En años sucesivos, y sobre todo a partir de 1950, la Jacetania sufrirá una pérdida de población generalizada en más del 90 por 100 de los municipios que la integran, situándose los índices de despoblamiento entre el 50 y el 75 por 100 del total de habitantes, y en algunos como Borau y Fago, alcanzando índices verdaderamente alarmantes próximos al 100 por 100.

Este proceso migratorio se ha llevado a cabo de forma paulatina. En muchos casos, previamente al salto definitivo a otras provincias desarrolladas, se produce la instalación de la población activa rural en Sabiñánigo que absorbe cantidad de mano de obra, o Jaca, que convertida en ciudad de servicios de la zona ejerce su papel rector del turismo estival e invernal, desarrollado de manera preferente en los valles de Aísa, Canfranc y Sallent de Gállego, que se han convertido así en núcleos de atracción para la población de los altos valles.

De esta forma, Sabiñánigo se ha visto favorecido por un incremento de población del 100 por 100 al 200 por 100 de sus habitantes, crecimiento astronómico que en cierto modo impulsa/recibe los beneficios del eje Sabinánigo-Jaca, ciudad que ha experimentado un auge poblacional cuyo indice se sitúa entre el 25 por 100 y el 50 por 100. La promoción de las pistas de esqui de Candanchú, Formigal, Panticosa y Astún se apoya precisamente en el eje señalado que aúna industria y servicios y mantiene en el territorio una densidad de población de 9 hab./ $\mathrm{km}^{2}$ con un índice de dispersión del 81 por 100.

Sobrarbe y Ribagorza que alcazarán su máximo poblacional en 1920 con 19.519 habitantes y $\mathbf{2 8 . 7 8 4}$ habitantes respectivamente, presentan un cuadro parecido en cuanto a la tendencia decreciente de efectivos de población y actividad económica se refiere. Las pésimas comunicaciones, la carencia de industria y de ciudades intermedias tipo Jaca y Sabiñánigo y la gran distancia que separa estas zonas de la capital de la provincia y otros centros industrializados, acentúa por una parte el proceso de despoblación pero por otra, provoca un freno natural a la colonización urbana acelerada 
en los últimos quince años, consiguiéndose por tanto de manera indirecta una defensa del paisaje. Sobrarbe es de las tres comarcas naturales que citamos, la más castigada por la emigración sobre todo en las zonas altas del interfluvio Ara-Cinca (Puértolas, Fanlo, Valle de Solana), donde el índice de pérdida poblacional se aproxima al 85 por 100 del total de habitantes, y en las franjas limitrofes de Sobrarbe por el Oeste y Suroeste. Estas zonas han sufrido una despoblación brutal superior al 50 por 100 que ha conducido a que municipios enteros, incluso valles enteros, estén vacíos hoy de tal forma que Sobrarbe arroja una densidad de $3 \mathrm{hab} . / \mathrm{km}^{2}$ con un índice de dispersión que se sitúa en el 106 por 100 . La distribución de estos habitantes en los $2.084 \mathrm{~km}^{2}$ de Sobrarbe no es homogénea, dándose los mayores núcleos y las densidades más altas cerca de las principales vias de comunicación que salvo la que cruza La Fueva, paralela a Sierra Ferrera y peña Montañera, siguen el curso de los ríos Ara y Cinca. Como consecuencia del envejecimiento de la población, la edad media de los matrimonios es bastante más elevada que la de la provincia y los nacimientos vienen siendo cada vez más escasos, contabilizando en los últimos diez años una tasa de 4 por 1.000 nacimientos, mientras que la global de España se sitúa en 20 por 1.000. La mortalidad se sitúa en los últimos años en un indice próximo al 12,8 por 1.000 habitantes, mientras que en España se halla alrededor del 8. (M. T. RUBIO BENITO y P. MUNUERA GIL, 1979).

El caso de Ribagorza es menos espectacular por cuanto que la mayor parte de sus municipios mantienen estacionaria prácticamente la pérdida de población en índices que se sitúan entre un 25 y un 40 por 100 . Solamente el límite fronterizo con Cataluña pierde más de 50 por 100 de su población sobre todo a partir de 1960; la densidad por $\mathrm{km}^{2}$ es de seis habitantes con un índice de dispersión de 129 por 100. Estas mismas posiciones se observan en las tasas de incremento de poblaciones que se mantienen más o menos estables hasta 1950 en que se produce una pérdida de población próxima al 50 por 100 .

En las comarcas de Sobrarbe y Ribargorza, más de la mitad de la población activa se dedica al sector primario, prácticamente estacionario, en manos de una población altamente envejecida que alcanza una edad media de 65 años, hecho que queda expresivamente de manifiesto en las pirámides de población de estos municipios representativos de las tres zonas comentadas. Más que pirámides se trata de cremalleras melladas o carentes de representación en la mayor parte de sus brazos de población sin capacidad de regeneración en corto espacio de tiempo, como ha ocurrido en el valle del Guarga en lo que va de siglo: su total extinción. 
Este proceso, como el de la despoblación de la Sierra de Guara y de todas las Sierras Exteriores y como tantos otros, es de lenta decadencia desde principios de siglo, incluso antes, de unos pueblos aislados por falta de comunicaciones, con una economia orientada hacia el autoabastecimiento y sin posibilidad de adaptarse a una economía de mercado que obliga a la especialización de la producción. Con la primera emigración se rompe el círculo cerrado de relación que mantenía el 99 por 100 de los habitantes, como reflejan el inmovilismo de los apellidos repetidos muchas veces, y desaparecen oficios típicos como cuchareros, vigueros, sogueros, guarnicioneros, herreros... etc.

Una vez roto este equilibrio de relaciones, la sociedad se deshace. Pero el verdadero problema radica en que como consecuencia de la regresión demográfica en algunos casos y de la extinción de población total en otros, se produce un abandono de tierras de labor considerable, con el progresivo aumento del saltus sobre el ager, la destrucción de los bancales, el arrastre de tierras por escorrentía y, en definitiva, la degradación del paisaje acentuada por el abandono de la infraestructura viaria, las casas, etc...

\section{¿Se trata por lo tanto de un proceso reversible o irreversible?}

Creemos que la revalorización del espacio rural sólo es posible mediante desarrollo endógeno, contando para ello con la existencia de una población que no se halle en situación límite de no poder asegurar el relevo generacional y que pueda adaptarse a otra actividad como la de servicios turísticos del sector terciario y espacios de ocio. 


\section{BIBLIOGRAFIA}

BIESCAS, J. A. (1977): Introducción a la economía de la región aragonesa. Alcrudo-Zaragoza.

Calvo Palacios, J. L. (1981): "La organización del espacio aragonés». Geografía de Aragón. Tomo I. Guara, Zaragoza. pp. 271-310.

INE: Censos de la población de España.

INE: Evolución de la población española en el período 1961-1978. Madrid 1980.

Molina lbánez, M. y Marín Jaime, J. M. (1981): Problemática socioeconómica aragonesa. CAE, FCEE. Zaragoza.

QUINTANA, F. (1981): Demografía y crecimiento económico aragonés en el período 1900-1936. CAE, FCEE. Zaragoza.

Rodriguez Osuna, J. (1985): Población y territorio en España. Siglos XIX y XX. Espasa Calpe. Madrid.

RuBIO BENITO, M. T. (1983): "El Somontano de Barbastro", pp. 247265. “El Cinca Medio», pp. 295-317. Geografía de Aragón. T. III. Guara. Zaragoza. "La Litera», pp. 11-22. «El Bajo Cinca», pp. 31-39. Geografía de Aragón. T. VI. Guara. Zaragoza.

Rubio Benito, M. T. y Castelló Puig, A (1986): «Utilización del espacio en el Valle de Tena", en: Actas de las I Jornadas de utilización del espacio en áreas de montaña. Cometa. Zaragoza, pp. 227-255.

Rubio Benito, M. T. y Munuera GiL, P. (1979): «La propiedad pública, factor condicionante del desarrollo económico de los altos valles pirenaicos oscenses", en Actas del VI Coloquio de Geógrafos españoles. AGE. Palma de Mallorca. 


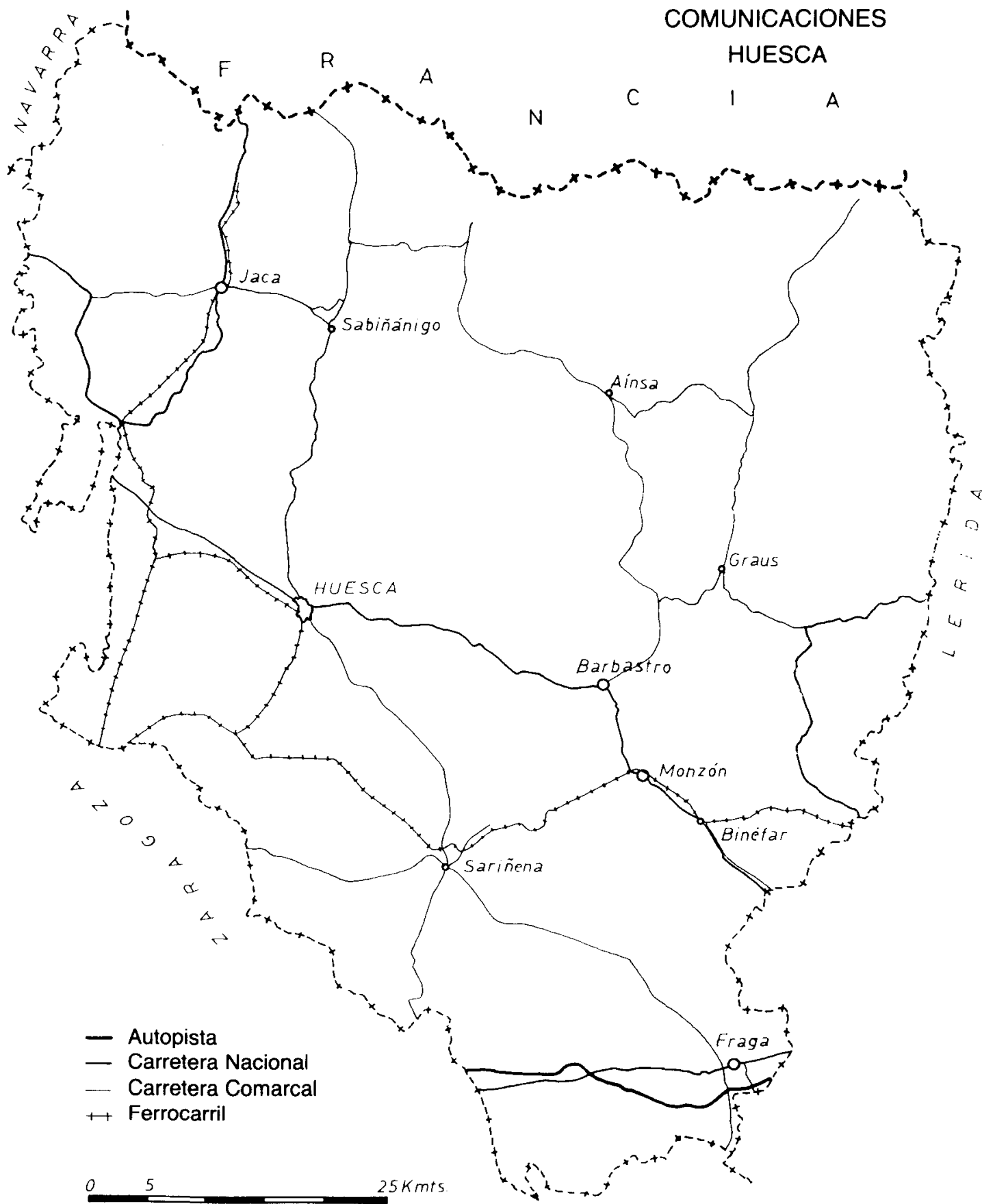


POLIGONOS INDUSTRIALES

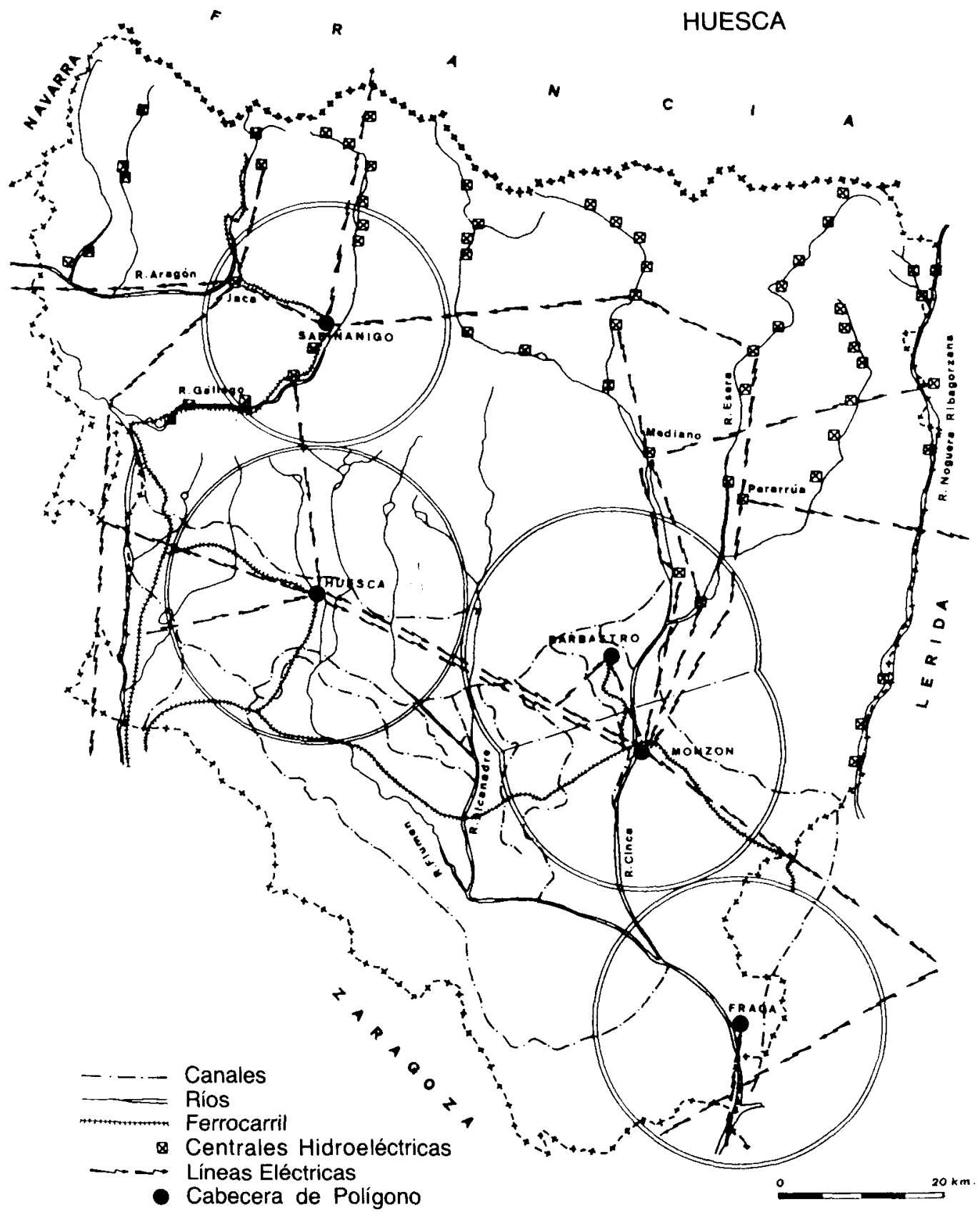


DENSIDAD DE LA POBLACION 1981 HUESCA

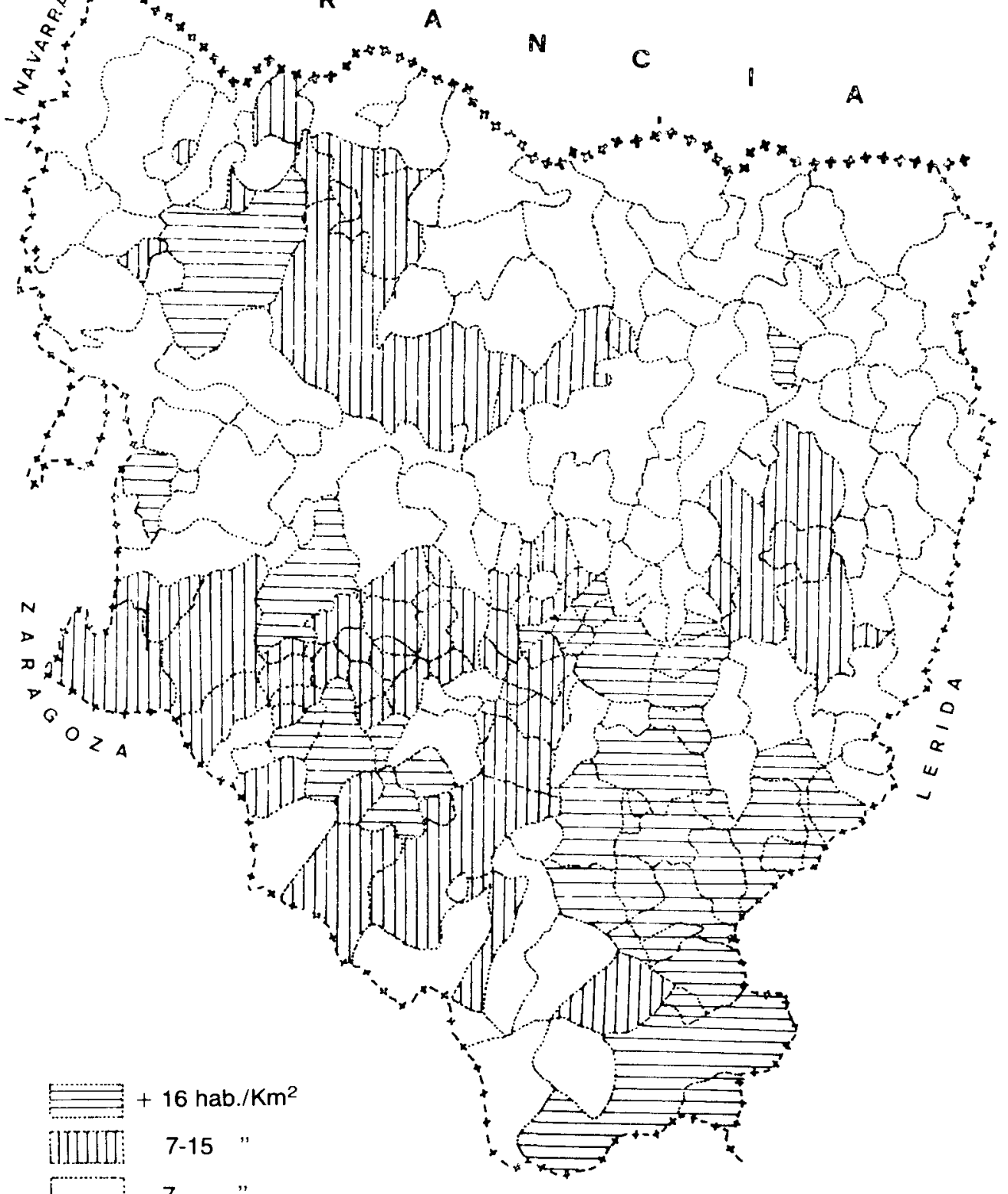
- 7

Fuente: Censos INE. Elaboración propia. 